\title{
La política de seguridad en México entre 2012 y 2018 . De la confrontación a la prevención del delito
}

\section{The security policy in Mexico between 2012 and 2018. From confrontation to crime prevention}

doi: https://doi.org// 0.32870/eees.v27i77.7082

Gerardo Hernández

\section{Resumen}

El presente artículo analiza la política de prevención del delito implementada durante la administración del presidente mexicano Enrique Peña Nieto (20 I2-20 I8).Se utilizan las teorías del nuevo institucionalismo y de las fallas de gobierno para argumentar que la falta de resultados en la política de seguridad en el país en dicho sexenio se debió a tres factores fundamentales: los problemas inherentes al suministro burocrático, los problemas de implementación y la descentralización política en el país. Se concluye que dicha falta de resultados evidenció, por un lado, la cooperación de los Gobiernos subnacionales en políticas prioritarias del Gobierno central, como la aprobación de las llamadas reformas estructurales, y por otro, la poca coordinación de los distintos niveles de gobierno ante la violencia e inseguridad, que terminó atomizada al final del periodo.

Palabras clave: seguridad pública, política pública, prevención del delito, nuevo institucionalismo, fallas de Gobierno.

\begin{abstract}
This article analyzes the crime prevention policy during the administration of Mexican President Enrique Peña Nieto (20 I2-20 I8). Theories of New Institutionalism and Failures of Government are used to argue that the lack of results in the sexennium about the security policy was due to three fundamental factors: The problems inherent to the bureaucratic supply, the problems of implementation, and the political decentralization in the country. The conclusions are that such lack of results shows, on the one hand, the cooperation of subnational governments in priority policies of the central government, such as the approval of the so called structural reforms and, on the other, the lack of coordination about violence and insecurity, problems that ended up atomized at the end of the period.
\end{abstract}

Keywords: Public security, public policy, prevention of crime, New Institutionalism, Failures of Government.

\footnotetext{
- Investigador del Instituto de Investigaciones Sociales de la Universidad Autónoma de Baja California (UABC), México. ORCID: https://orcid.org/0000-0003-2803-6905

ghernandez48@uabc.edu.mx

Fecha de recepción: 16 de enero de 2019. Fecha de aceptación:04 de julio de 2019.
} 
Introducción'

Este artículo analiza la estrategia de seguridad diseñada e instrumentada por la administración del presidente mexicano Enrique Peña Nieto (2012-2018). Es producto de una investigación cuya pregunta fue ¿por qué si la política de seguridad 2012-2018 planteó un enfoque diferente al de la administración federal antecesora, esta vez basado en la prevención del delito, dicha nueva política no logró los objetivos planteados, y en cambio se dio en el país una atomización de la inseguridad al final del periodo de Gobierno peñanietista?

Como hipótesis, se planteó que la política de prevención de la administración de Peña Nieto arrancó con fuerza al inicio de su gestión, pero luego se debilitó por los llamados problemas inherentes al suministro burocrático. Específicamente, se llevaron a cabo cuatro cambios del responsable de la política de prevención en el país, todos con distintos perfiles. Esto, a su vez, generó problemas de implementación, sumados al contexto de descentralización política en el territorio que se manifestó en la falta de presencia del Estado en diversas entidades federativas, cuyos gobernadores, conscientes de la fragmentación del poder político, dejaron que el costo de la inseguridad en sus demarcaciones lo asumieran el Gobierno federal y la sociedad. En este mismo contexto, los intereses particulares del Gobierno federal 2012-2018 se concentraron en la aprobación e implementación de las reformas estructurales en materia económica.

I. Se extiende un agradecimiento por su valioso apoyo a las estudiantes de la Licenciatura en Administración Pública y Ciencia Política de la Universidad Autónoma de Baja California, Alejandrina Oropeza Noriega y Jocelyn Hopper Andrade, para la realización de este artículo. La primera colaboró en su calidad de becaria del Programa para el Desarrollo Profesional Docente (PRODEP) y la segunda como prestadora de servicio social, junto al estudiante de la Licenciatura en Sociología por la misma universidad, Humberto Yañez Ruiz. 
Para la comprobación de la hipótesis antes señalada, el estudio se construyó sobre un modelo explicativo.

El presente artículo se divide en tres apartados. En el primero, se presentan los referentes de las teorías del nuevo institucionalismo y de las fallas de gobierno. Estas teorías permiten analizar el diseño e implementación de una política pública, asumiéndola como una institución en la cual intervienen diversos actores que impactan en sus resultados a corto y largo plazo. En el segundo y el tercer apartado, se analiza el contexto político en el que se diseñó el Programa Nacional para la Prevención Social de la Violencia y Delincuencia (PNPSVD), así como los resultados del mismo, en los que se identifican los problemas inherentes al suministro burocrático y de implementación. En el cuarto y quinto apartado, y considerando los tres anteriores, se explica por qué la descentralización es una variable que ayuda a comprender que cuando una política pública no tiene bases sólidas, aunada a intereses particulares, termina fragmentada en sus objetivos y da espacios para que los problemas que atiende se agudicen. Al final, se presentan las conclusiones.

La investigación se respalda con datos del Secretariado Ejecutivo del Sistema Nacional de Seguridad Pública (SEsnsp), del Observatorio del Gasto Público de la Secretaría de Gobernación (Segob), de la Encuesta Nacional sobre Percepción de Inseguridad Ciudadana en México, del Instituto Nacional de Estadística y Geografía (INEGI), así como de seguimiento a fuentes hemerográficas.

\section{El nuevo institucionalismo y las fallas}

de gobierno en las políticas públicas

Los aportes de Lasswell (2007), Lindblom (2007), Garson (2007), Parsons (2007), Franco Corzo (2013) y Merino (2013), por mencionar algunos, han sido fundamentales 
para entender que el objetivo de las políticas públicas es mejorar la toma de decisiones en el ámbito de lo público, previa generación de información con base en diagnósticos y análisis de factibilidad. De esta manera, ante problemas que surgen en los contextos sociales, económicos y políticos, una política pública generalmente constituye la respuesta a una demanda social. Por otro lado, también puede ser la anticipación de las autoridades gubernamentales para atender y desactivar posibles problemas sociales que podrían detonar en el futuro de un modo más peligroso. Esto no quiere decir, no obstante, que a veces las políticas mismas no representen dificultades que provoquen demandas y produzcan exigencias (Pasquino, 2011, p. 270).

En este mismo sentido, Peters (citado en Pérez Sánchez, 2005 , p. 53), plantea que política pública es "el conjunto de actividades de las instituciones de Gobierno, actuando directamente o a través de agentes, y que van dirigidas a tener una influencia determinada sobre la vida de los ciudadanos". Para que una política sea considerada como pública, debe haber sido generada en el marco de los procedimientos, instituciones y organizaciones gubernamentales. Esto quiere decir que se trata de estrategias de actuación en cuya configuración las organizaciones públicas desempeñan un papel clave, aunque la participación de las mismas no sea exclusiva. Lo anterior se menciona porque muchas políticas son implementadas con colaboración o a través de organizaciones privadas (Pérez Sánchez, 2005, p. 52).

También es importante señalar que cuando no se alcanzan los objetivos de la política pública, es inevitable cuestionar ¿por qué fallan las políticas y por qué pareciera que los Gobiernos, ante ciertos problemas públicos, quedan cortos en su capacidad de respuesta? Como réplica a este interrogante, se pueden señalar dos perspectivas que se relacionan entre sí. La primera tiene que ver con la teoría clásica sobre lo que son las políticas públicas, y la segunda 
retoma lo anterior pero desde el enfoque del nuevo institucionalismo y de las fallas de gobierno.

La primera perspectiva, retomando a Lindblom y Parsons, plantea que los decisores tienen poco control de su entorno, por lo que deben realizar análisis marginales a partir de alternativas mínimas. De esta forma, el decisor selecciona la política que ofrece un incremento de valor marginal respecto a las políticas previas o a las políticas alternativas (Parsons, 2007, pp. 201-225). Asimismo, dentro de este proceso, es necesario considerar las elecciones, las burocracias, los partidos, los políticos y los grupos de interés que estructuran y distorsionan el proceso de las políticas públicas (Parsons, 2007, p. 56).

En cuanto a la segunda perspectiva, el nuevo institucionalismo como herramienta de análisis hace hincapié no sólo en los atributos formales de las instituciones, sino también en la distribución informal del poder, en las actitudes y en el comportamiento político, es decir, sugiere colocar la mirada en los deseos y comportamientos concretos, observables, de grupos e individuos (Romero, 1999, p. 9).

Desde este enfoque, se destaca la importancia de la racionalidad individual, limitada en general por un conjunto de instituciones que permiten obtener la mayor rentabilidad para el grupo que plantea las modificaciones a las reglas (López Sandoval, 2010, p. 303). Por institución, y de acuerdo con North (1993), se entiende a

[...] las reglas del juego de una sociedad o, más formalmente, son las limitaciones ideadas por el hombre que dan forma a la interacción humana. Por consiguiente, estructuran incentivos en el intercambio humano, sea político, social o económico. Las instituciones reducen la incertidumbre por el hecho de que proporcionan una estructura a la vida diaria. (pp. 13-14) 
Al mismo tiempo, uno de los grandes aportes del nuevo institucionalismo aplicado al estudio de las instituciones es su utilidad para el análisis en la creación o modificación de reglas, pues permite entender la búsqueda de la maximización de la rentabilidad por parte de los individuos. Esto se logra considerando a una institución como un contexto estratégico. De esta manera, un grupo de agentes o individuos dentro de la estructura gubernamental puede diseñar un conjunto de instituciones (las cuales, al menos en el discurso, van a beneficiar a la sociedad), pero con consecuencias opuestas a las iniciales en el proyecto.

Con esto, Colomer (1991) señala que se reconoce que los individuos forman parte de los colectivos, pero también buscan obtener sus objetivos mediante el establecimiento de sus propias estrategias y decisiones. En palabras de Buchanan y Tullock, los individuos son los únicos responsables de la determinación de la acción del grupo, así como de la acción privada (1993, p. 22). Lo anterior está alejado de la creencia de que los individuos poseen bondad innata al momento de tomar decisiones, o que los acuerdos son producto de una mera clarificación o diálogo intelectual en el terreno moral (Colomer, 1991).

Todos estos aportes ayudan a entender la trayectoria que toman las políticas públicas, y sobre todo aquello que Parsons (2007, p. 358), Medellín (2004, pp. 23-26) y Real Dato (2005, pp. 77-107) han expuesto, es decir, que las maniobras del Gobierno están subordinadas por el cúmulo de intereses, tensiones y conflictos presentes en las organizaciones y la sociedad. Los actores como los partidos políticos, las asociaciones empresariales, de trabajadores y demás formas de organización política emergen como instancias de negociación de intereses individuales, en un escenario en donde el Gobierno también participa.

Este panorama también ha sido expuesto por Agudo Sanchíz (2015, pp. 123-146), quien señala que son las prácticas 
sociales las que realmente sostienen a los modelos de las políticas, aun cuando se asuma que los segundos generan las primeras. El mismo autor engloba lo ya expuesto, y plantea que las diversas etapas del proceso de formulación y evaluación de las políticas públicas pueden interpretarse entonces como un campo político constituido por la confrontación entre individuos y grupos que actúan desde sus dispares posiciones de poder e influencia.

Por tal motivo, cuando el Gobierno falla en la resolución de los problemas, se tiene que observar lo antes expuesto, así como el diseño de las políticas y el contexto sociopolítico. A esto, Weimer y Vining (2005, pp. 163-187) lo denominan fallas de Gobierno, e identifican al menos tres componentes que dificultan una correcta implementación de políticas públicas: problemas inherentes al suministro burocrático, problemas inherentes a la descentralización y el problema de la implementación.

Por problemas inherentes al suministro burocrático se entiende a los vinculados con labores que los Gobiernos a menudo realizan -por ejemplo, la instrumentación de leyes o reformas-para hacer frente a una serie de problemáticas. Los encargados de implementar las políticas, a diferencia de lo que sucede en las empresas privadas, no requieren pasar una prueba de mercado para sobrevivir (difícilmente hay costos que asuman), esto debido a que el bienestar social que sus acciones persiguen depende en gran medida de la diligencia y las motivaciones de los representantes. En otras palabras, la propia naturaleza de los organismos públicos hace difícil la vigilancia sobre las acciones, y su ineficiencia es probable (Weimer y Vining, 2005, p. 179).

$\mathrm{Al}$ referirse a los problemas de la implementación, los autores plantean que no sólo por el hecho de diseñar una política pública esta siempre logrará los resultados previstos. En este sentido, se argumenta que una política basada en una teoría incorrecta puede producir consecuencias 
no deseadas. Incluso, partir de un diseño efectivo (teoría correcta) sólo es una cuestión necesaria, pero no suficiente, para una correcta implementación.

Lo anterior se debe a que los Gobiernos centrales deben confiar en los niveles inferiores de Gobierno para la realización de las acciones. Aun cuando el Gobierno central goza de autoridad nominal sobre las unidades gubernamentales de nivel inferior, se enfrenta al problema de la vigilancia de las múltiples jurisdicciones, que pueden tener sus propios intereses. Asimismo, la implementación de políticas que requieren la cooperación de los Gobiernos de nivel inferior se vuelve aún más difícil cuando los centrales no tienen la autoridad para coaccionar. De esta manera, y con el fin de inducir a la cooperación con éxito, el Gobierno central debe ofrecer recompensas suficientes para garantizar el cumplimiento voluntario (Weimer y Vining, 2005, pp. 187-188).

En lo referente a los problemas inherentes a la descentralización, se expone que para instrumentar políticas hay que considerar que existen ramas separadas e independientes del Gobierno -Poderes Ejecutivos, Legislativos y Judiciales-. Además, algunas funciones son ejercidas por el Gobierno central y otras son reservadas a los Gobiernos estatales y locales. Asimismo, dentro de las ramas ejecutivas de estos distintos niveles de gobierno, los actores públicos, por ejemplo los gobernadores, a menudo disfrutan de una discreción considerable sobre la política y la administración (Weimer y Vining, 2005, pp. 187-188).

Los componentes expuestos también se relacionan con la complejidad de lo que Peters (2010) ha llamado the shadow of hierarchy. Esto quiere decir que el Gobierno central tiene que delegar autoridad a diversos actores sociales -en los que se incluye a los Gobiernos subnacionales-y redes que intervienen en las políticas públicas. No obstante, cuando los objetivos de las políticas públicas se desvían, el Estado debería tener la jerarquía para retomar el control y la cen- 
tralización de la implementación de las mismas. Dicho esto, el propio Peters (2010, p. 4) advierte que en los sistemas federales el proceso de mantener la jerarquía del Estado se complejiza debido a que los Gobiernos subnacionales apelan a la autonomía.

Lo anterior ayuda a entender lo que advirtió Lowi (citado en Ingram, De León, y Schneider, 2016, pp. 183-184), es decir, que los analistas de políticas públicas han dejado de lado los grandes cambios que está sufriendo la democracia y el impacto de esto para las políticas mismas. Por esta razón, la descentralización es una variable que invita a ser considerada cuando se analizan políticas públicas. Como señalan Ingram et al. (2016, pp. 175-182), las políticas públicas no están alcanzado sus objetivos (involucrar, habilitar e inspirar la participación ciudadana) en las democracias más arraigadas, y mucho menos en Estados que han transitado a la democracia y han pasado por un proceso de descentralización política, como es el caso de México.

\section{Diseño y fallas de la prevención social}

de la violencia y la delincuencia

Después de las elecciones presidenciales de 2012 en México, el ganador de la contienda, el candidato de la coalición entre el Partido Revolucionario Institucional (PRI) y el Partido Verde Ecologista de México (PVEM), Enrique Peña Nieto, dejó clara, al menos en el discurso, la postura que tomaría su estrategia de seguridad, que buscaría establecer distancia con respecto a la administración de su antecesor, el expresidente Felipe Calderón (2006-2012).

En una entrevista con The Washington Post, Peña Nieto declaró: "Después de que asuma la Presidencia en diciembre próximo, el nuevo mandatario no medirá el éxito por el número de cabecillas del narcotráfico capturados o eliminados, o por los montos confiscados de cocaína, medidas que 
son populares entre funcionarios de la DEA o en el Congreso de Estados Unidos" (Hernández, 2012).

No obstante, esto no significó que la política de seguridad tendría un cambio realmente radical respecto a la estrategia presentada por su antecesor Calderón. La diferencia estaría en la reducción de la violencia a través de la prevención del delito. En el Plan Nacional de Desarrollo 2013-2018, una de las metas se nombró México en Paz, y mediante ella se atendería la prevención del delito como uno de los problemas antes dejados de lado (Diario Oficial de la Federación, 2013). De manera concreta, se persiguió "Reducir la violencia y recuperar la paz y la tranquilidad de las familias mexicanas. En particular, disminuir los indicadores relacionados con homicidios, secuestros y extorsiones" (Diario Oficial de la Federación, 2013).

Una de las ventajas de esta estrategia estaría en la coordinación, esto considerando que uno de los reclamos del expresidente Calderón fue la falta de apoyo de todos los agentes en cuanto a su involucramiento para el cumplimiento de las reglas creadas para combatir al crimen organizado (Hernández, 2015, pp. 159-187). El presidente Peña Nieto mencionó que en su Gobierno habría coordinación entre fuerzas federales y Gobiernos estatales, así como que las instituciones de seguridad no competirían entre ellas (Presidencia de la República, 2013).

Este discurso de coordinación era posible debido a que el PRI, después de la elección federal de 2012, mantenía el Gobierno de la mayoría de las gubernaturas del país (diecinueve de treinta y dos), así como una mayoría, junto a su coalición con el PVEM, en el Congreso de la Unión: en la Cámara de Diputados, el PRI obtuvo en 2012 el 43\% de los escaños y el PVEM el 5\% (Cámara de Diputados, 2015); asimismo, en la Cámara de Senadores, el PRI obtuvo el 42\% y el PVEm el 5\% (Cámara de Senadores, 2015). 
Este escenario político también favoreció la retórica conciliadora del Ejecutivo federal, quien puso de lado el tema de la inseguridad para favorecer la negociación -con el Poder Legislativo y los Gobiernos subnacionales- de la aprobación de las llamadas reformas estructurales: reforma energética, reforma en materia de telecomunicaciones, reforma en competencia económica, reforma financiera, reforma hacendaria, reforma laboral, reforma educativa, reforma político-electoral y reforma en materia de transparencia. ${ }^{2}$

Es importante señalar que el resultado del uso de estas tácticas retóricas, en un primer momento, fue políticamente significativo, tanto en aprobación del Gobierno federal -en 2012 , la aprobación del presidente fue de $53 \%$ y para 2013 de 57\% (Leyva, 2017) - como en reconocimiento a nivel internacional por los cambios estructurales realizados por la administración que iniciaba. ${ }^{3}$

En este contexto, la política de seguridad 2012-2018 se basó en diez estrategias con las cuales, de acuerdo con el propio Gobierno federal, se lograría recuperar la paz entre los mexicanos: 1) prevención del delito y reconstrucción del tejido social; 2) justicia penal eficaz; 3) profesionalización y fortalecimiento de los cuerpos de policía; 4) transformación del sistema penitenciario; 5) promoción y articulación de la participación ciudadana; 6) cooperación internacional; 7) información que sirva al ciudadano; 8) coordinación entre autoridades; 9) regionalización; y 10) fortalecimiento de inteligencia (Presidencia de la República, 2015a).

2. El 2 de diciembre de 2012, el llamado Pacto por México fue firmado por las principales fuerzas políticas del país con el fin de dar inicio a un proceso de negociación y aprobación de cada una de las reformas estructurales señaladas. 3. En 20l4, la revista Time dedico su portada internacional al presidente Enrique Peña Nieto, bajo el titular "Salvando México". 


\section{Programa Nacional para la Prevención}

Social de la Violencia y Delincuencia

Como se dijo, la política de seguridad para el periodo peñanietista tendría como base la prevención del delito, lo cual se materializó en el PNPSVD. En el mismo sentido, el presidente Enrique Peña Nieto declaró: "estoy convencido que el delito no sólo se combate con la fuerza. Es imprescindible que el Estado emprenda un esfuerzo integral para reconstruir el tejido social" (Presidencia de la República, 2015b).

Así, el PNPSVD tuvo cinco objetivos particulares: 1) incrementar la corresponsabilidad y participación ciudadana; 2) reducir la vulnerabilidad ante la violencia y la delincuencia en poblaciones prioritarias; 3) generar entornos que favorezcan la convivencia; 4) fortalecer capacidades institucionales para la prevención social; y 5) asegurar una coordinación efectiva entre los tres órdenes de gobierno (Diario Oficial de la Federación, 2014a).

El plan se elaboró con base en una subcategoría del concepto de seguridad humana, es decir, la seguridad ciudadana. Desde este enfoque, se buscó la participación ciudadana en la construcción de ambientes seguros a través de la prevención, además de entenderse la seguridad como un derecho humano que debe ser garantizado por el Estado pero que, a su vez, debe ser coproducido por todos los actores sociales (Diario Oficial de la Federación, 2014a).

El diagnóstico del PNPSVD planteó seis factores de riesgo asociados a la violencia y delincuencia: individuales, familiares, grupales, educativos, comunitarios y sociales y culturales. De igual manera, se estableció trabajar sobre los problemas específicos de embarazo temprano; consumo y abuso de drogas legales e ilegales; ambientes familiares deteriorados o problemáticos; deserción escolar; falta de oportunidades laborales, informalidad y desocupación; capital social debilitado y participación ciudadana incipiente; 
entornos de ilegalidad; espacios públicos para la convivencia insuficiente y deteriorada; y marginación y exclusión social.

Asimismo, los objetivos estarían focalizados en cincuenta y siete demarcaciones, las cuales, de acuerdo al PNPSVD, tenían

altas tasas delictivas y condiciones que constituyen factores de riesgo para el surgimiento o permanencia de distintos tipos de violencia, por lo que se requiere tanto la prevención de las causas como la atención de los efectos de las violencias y la delincuencia en el corto y mediano plazo. (Secretaría de Gobernación, 20 I3, p. 24)

No obstante, no todas las cincuenta y siete demarcaciones seleccionadas coincidieron con el criterio de la intervención focalizada, es decir, no todas tenían altas tasas delictivas y no imperiosamente estas constituían factores de riesgo para el surgimiento de distintos tipos de violencia.

Lo anterior se menciona debido a que no se consideraron municipios, delegaciones o zonas metropolitanas que podrían haber sido focos de surgimiento de algún tipo de violencia. Por ejemplo, se puede señalar que quedaron fuera un gran número de municipios del estado de Michoacán que sufrieron la confrontación de grupos de autodefensas y del crimen organizado. Tampoco se consideraron los municipios de Guerrero, lugar donde habían surgido las autodefensas durante la administración del presidente Felipe Calderón.

Lo señalado hace patente los problemas metodológicos de las bases del PNPSVD. Por ejemplo, del estado de Chihuahua, se seleccionaron los municipios de Chihuahua y Juárez, pero no se consideró la información que la Segob emitió el 29 de mayo de 2014, en la cual se especificó que la entidad tenía nueve municipios con mayor ambiente de violencia: Aldama, Allende, Bocoyna, Camargo, Cusihuiriachi, Julimes, Matamoros, Meoqui y San Francisco del Oro (Diario Oficial de la Federación, 2014b). 
También, el plan seleccionó al municipio de Othón P. Blanco en Quintana Roo en lugar de Chalco en el Estado de México, cuando el primero tuvo una cifra de novecientas noventa y una defunciones en 2012 y Chalco en el mismo año registró 1306 muertes (Instituto Nacional de Estadística y Geografía, 2015). Otro elemento que debió considerarse es el tamaño poblacional: Chalco cuenta con 310130 habitantes, y Othón P. Blanco con 244553 (Instituto Nacional de Estadística y Geografía, 2015). ${ }^{4}$

Un caso más fue la selección de Mérida, Yucatán, como una de las demarcaciones prioritarias, mientras no se consideraron municipios de Michoacán como Buenavista. Esto se menciona porque el índice de paz 2015 de la Organización de las Naciones Unidas (ONU) señaló que Yucatán se ubicó entre los cinco estados más pacíficos del país, junto a Hidalgo, Querétaro, Campeche y Tlaxcala (Instituto para la Economía y la Paz, 2015).

De igual manera, se dio el caso de demarcaciones con características similares y que fueron elegidas una por encima de otra, como pasó con la elección de Calpulalpan, Tlaxcala, por encima de Buenavista, Michoacán: la primera registró doscientas treinta y siete muertes en 2012, mientras que en la segunda se contabilizaron doscientas quince; en cuanto a su tamaño poblacional, en 2014 Calpulalpan contaba con 44807 habitantes y Buenavista con 42234 (Instituto Nacional de Estadística y Geografía, 2015). A nivel entidad federativa, en el mismo año de 2014, Tlaxcala registró trescientos sesenta homicidios y siete secuestros, mientras que Michoacán tuvo 2365 homicidios y ochenta y tres secuestros (Secretariado Ejecutivo del Sistema Nacional de Seguridad Pública, 2019, p. 32).

4. Además de esto, el 28 de mayo de 2015, Policía Federal, Ejército y Marina, en coordinación con las autoridades de la Ciudad de México, tuvieron que iniciar un operativo en Tláhuac y Chalco para combatir los delitos de alto impacto (secuestros, extorsiones, robos, homicidios y violaciones) (Ruiz, 20I5).

\section{6}




\section{Los problemas inherentes al suministro}

burocrático y de implementación

Lo expuesto conlleva analizar y explicar por qué los objetivos en torno a seguridad no se alcanzaron al término de la administración de Peña Nieto. Como se planteó en la hipótesis, se identifican en la misma problemas inherentes al suministro burocrático y de implementación, sobre todo al considerar que el Gobierno peñanietista partió de un diagnóstico y de la experiencia del Gobierno federal previo. En cuanto a las fallas de Gobierno identificadas, también se relacionan con lo planteado por Weimer y Vining (2005, pp. 179-181), es decir, que crear políticas mediante una agenda política mediática puede desalentar la cuidadosa evaluación de alternativas al problema que se busca resolver. Esto se señala porque la propuesta inicial del Ejecutivo federal de Peña Nieto fue, como se dijo antes, resolver el problema de la inseguridad atendiendo y reconstruyendo el tejido social.

Para llevar a cabo el objetivo de la prevención social de la violencia, en 2013 se creó la Subsecretaría de Prevención y Participación Ciudadana, institución encargada de implementar el PNPSVD, que a su vez necesitaba de la coordinación de los tres órdenes de gobierno para atender el tejido social de las demarcaciones seleccionadas en el plan. No obstante, el hecho de que, antes de finalizar la administración federal, el PNPSVD y la misma subsecretaría encargada dejaran de tener relevancia en la agenda de gobierno evidenció dos fallas de la política implementada, vinculadas a los problemas inherentes al suministro burocrático y de implementación.

La primera falla se relaciona con los errores en la metodología, ya antes señalados, sobre la que se creó el programa emblema de la política de seguridad del periodo presidencial en cuestión. En otras palabras, al buscar abarcar todo el país, se dejaron de lado entidades federativas que reque- 
rían de intervenciones prioritarias en toda su extensión territorial (por ejemplo, Guerrero y Michoacán) y no sólo en algunas de sus demarcaciones.

Como segunda falla, y desde los referentes teóricos del nuevo institucionalismo, se ubica el factor político. Esto se menciona debido a que, como señalan Acemoglu y Robinson (2013, pp. 59-60),

son las instituciones políticas las que determinan cómo funciona el proceso político. Por ejemplo, las instituciones políticas de una nación determinan la capacidad de los ciudadanos de controlar a los políticos e influir en su comportamiento. Esto, a su vez, determina si los políticos son agentes de los ciudadanos, o si son capaces de abusar del poder que se les confía [...] y seguir sus objetivos personales en detrimento de los ciudadanos.

En el caso del PNPSVD, en cinco años (2013-2018), la Subsecretaría de Prevención y Participación Ciudadana tuvo cuatro subsecretarios: Roberto Campa Cifrián (2013-2015), Arturo Escobar y Vega (2015), Alberto Begné Guerra (2016-2017) y José Luis Stein Velasco González (2018). Cabe señalar que el perfil de cada subsecretario, salvo el caso de Roberto Campa Cifrían, no necesariamente estuvo relacionado a temas de seguridad o de prevención del delito (Tabla 1). 
Tabla 1. Perfiles de los subsecretarios de Prevención

y Participación Ciudadana (2013-2018)

\begin{tabular}{|c|c|}
\hline $\begin{array}{l}\text { Roberto Campa } \\
\text { Cifrián }\end{array}$ & $\begin{array}{l}\text { Abogado, político y empresario. Candidato a la } \\
\text { presidencia por el partido Nueva Alianza en 2006. Ha } \\
\text { ocupado diversos puestos en la administración pública, } \\
\text { como secretario ejecutivo del Sistema Nacional de } \\
\text { Seguridad Pública (2007-2008), subsecretario de } \\
\text { Prevención y Participación Ciudadana, subsecretario } \\
\text { de Derechos Humanos y secretario del Trabajo en la } \\
\text { administración federal 2012-2018. }\end{array}$ \\
\hline $\begin{array}{l}\text { Arturo Escobar } \\
\text { y Vega }\end{array}$ & $\begin{array}{l}\text { Abogado. Miembro del PVEM. Diputado federal y local } \\
\text { por el Distrito Federal. Consejero del PVEM ante el } \\
\text { Instituto Federal Electoral (IFE). Vocero nacional } \\
\text { del PVEM. En 2008, propuso la pena de muerte a } \\
\text { secuestradores. }\end{array}$ \\
\hline Alberto Begné & Abogado y académico. Exdirigente del Partido Social \\
\hline Guerra & Demócrata. \\
\hline José Luis & Extitular de Coordinación Ejecutiva de la Secretaría \\
\hline Stein Velasco & de Educación Pública y extitular de la Unidad de \\
\hline González & $\begin{array}{l}\text { Asuntos Internacionales de la Secretaría del Trabajo y } \\
\text { Previsión Social. }\end{array}$ \\
\hline
\end{tabular}

Fuente: elaboración propia.

Dicho esto, y también desde el nuevo institucionalismo, la creación de la subsecretaría, como un contexto estratégico, respondió más a intereses particulares de quienes controlaban el poder político que al hecho de buscar consolidar los objetivos que se perseguían con el PNPSVD. En otras palabras, los nombramientos se debieron más a coyunturas políticas que a la búsqueda de fortaleza para la prevención social del delito. ${ }^{5}$

5. En 20I5, Roberto Campa Cifrián fue nombrado secretario del Trabajo y en su lugar llegó Arturo Escobar y Vega, quien a la postre presentaría su renuncia. Cuando el presidente de la república sustituyó al secretario de Gobernación Miguel Ángel Osorio Chong, nombró en su lugar a Alfonso Navarrete Prida, quien llevó a cabo dieciocho cambios en toda la institución, incluida la Subsecretaría de Participación y Prevención Ciudadana.

Estado No.77 
Por ejemplo, el nombramiento de Arturo Escobar y Vega -miembro del PVEM y aliado del PRI en la elección presidencial de 2012- fue cuestionado por organizaciones civiles debido a que su perfil no tenía ninguna relación con la dirección de esa subsecretaría (Instituto Mexicano de Competitividad, 2015). En 2015, Escobar y Vega renunció al puesto debido a que la Fiscalía Especializada para la Atención de los Delitos Electorales (FEPADE) solicitó una orden de aprehensión en su contra (Méndez, 2016).

Aunado a los problemas inherentes al suministro burocrático y de implementación que se identifican, también está el hecho de que la subsecretaría quedó acéfala durante tres meses después de la renuncia de Arturo Escobar y Vega. Esto a su vez demostró el poco interés del Gobierno central para lograr los objetivos de la política pública de prevención.

Asimismo, y desde la perspectiva teórica, lo sucedido se puede explicar considerando que los agentes encargados de implementar políticas, a diferencia de lo que sucede en las empresas privadas, no tienen controles de calidad o pruebas de mercado que les representen costos para sus propios intereses, como perder el cargo si no fueran alcanzados los objetivos de una política pública.

Como se plantea en la hipótesis enunciada al inicio del artículo, el declive en la importancia del PNPSVD también se debió a los intereses particulares del Gobierno federal, el cual debió negociar, durante veinte meses al inicio del mandato, la aprobación de las reformas estructurales firmadas en el Pacto por México. En dicho periodo, se requirió tiempo y negociación por parte del Ejecutivo federal con los demás agentes políticos, incluidos los Gobiernos estatales, tiempo durante el cual el PNPSVD fue reduciendo su presupuesto, lo que no se detuvo hasta el final de la administración federal (Figura 1). 
Figura 1. Presupuesto del programa (2013-2018)

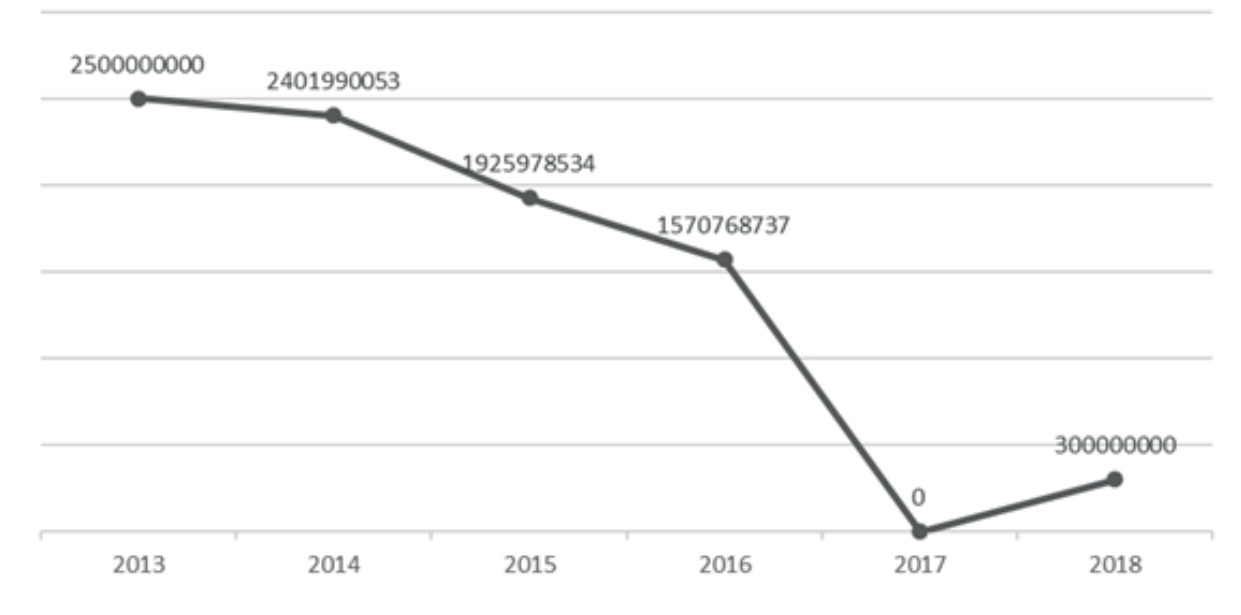

Fuente: elaboración propia con datos de Transparencia Presupuestaria (2018).

No obstante, para el Gobierno federal entre 2012 y 2018, al menos en el discurso y particularmente al inicio de la administración, una política de prevención para reconstruir el tejido social se alineaba con los intereses de dar a conocer en el plano internacional la agenda reformista del Ejecutivo federal: "la decisión del presidente no fue atacar al crimen organizado[,] sino prevenir sus causas [...] esa es la manera en que puede proyectar tanto a nivel nacional [como] internacional la percepción de seguridad[,] que es la manera en que nuestro Gobierno puede medir si está funcionando el programa" (Leal, 2015). En este mismo sentido, y como señala Franco Corzo (2013, p. 52), la idea de querer resolver un problema a través de una política pública puede quedar sólo en un decálogo de buenas intenciones si no se consideran la factibilidad presupuestal, socioeconómica, legal, social, política, ambiental y administrativa de las alternativas de acción.

El argumento teórico expuesto también se relaciona con los aportes de Cano y Rojido (2016, pp. 6-195), quienes plantean la complejidad que existe al analizar el impacto 
de los programas de prevención de la violencia en países como Brasil, Venezuela, Honduras, Jamaica, México (en específico, Ciudad Juárez) y Colombia. Los autores precisan que la gran mayoría de los programas de prevención de la violencia no cuentan con evaluaciones de impacto, y mucho menos evaluaciones de costo-beneficio metodológicamente rigurosas que permitan emitir un juicio concluyente sobre ellos. En este mismo sentido, los autores exponen que la mayoría de las iniciativas en la región bajo estudio cuenta con auditorías y evaluaciones de implementación a menudo realizadas por el propio personal que ejecuta el programa.

A lo dicho se suma la evaluabilidad reducida que tienen muchos programas por la falta de claridad de sus objetivos y componentes, esto debido a que se incluyen en los mismos un gran número de intervenciones de naturaleza diversa, de modo que hace difícil saber cuáles de ellas están provocando qué impacto. Este fenómeno se aprecia no sólo a nivel macro, sino también a nivel micro, es decir, incluso en los casos minoritarios en que existen evaluaciones de impacto, los resultados no siempre son concluyentes, y a menudo existen controversias sobre el grado de éxito alcanzado.

En México, estas áreas de incertidumbre, aunadas al diseño institucional de programas como el PNPSVD, son lo que ha permitido rentabilizar políticamente la retórica gubernamental de la lucha contra la violencia e inseguridad, aunque, como se ha expuesto hasta ahora, al final no se puedan medir los impactos positivos del plan. Cabe señalar que este tipo de estrategias de rentabilización del diseño institucional de programas enfocados a atender problemas sociales ya se ha documentado con antelación. Por ejemplo, López Sandoval (2007, pp. 12-208) analiza la relación positiva entre resultados electorales y el gasto social en el ámbito federal en el periodo 1994-2006, específicamente respecto a los programas Nacional de Solidaridad 
(PRONASOL), de Educación, Salud y Alimentación (PROGRESA) y Oportunidades.

Este tipo de escenarios, desde la perspectiva de Agudo Sanchíz (2017), también se presentaron con el PNPSVD. De manera específica, el objetivo del PNPSVD

[...] no es reducir la violencia y el delito, sino ofrecer la prevención como pantalla cosmética que permite a socios y aliados transnacionales minimizar escrúpulos y presiones en sus transacciones con México; todo ello al tiempo que se distribuyen recursos públicos mediante el esquema legitimador de la apertura al sector de las organizaciones de la sociedad civil, cuya participación se celebra entonces como un logro. (Agudo Sanchíz, 2017, p. 298)

\section{La descentralización democrática como variable para} entender las fallas de la prevención social de la violencia y la delincuencia

Lo expuesto llevó a que los grandes objetivos que perseguía la prevención social de la violencia y la delincuencia durante la administración federal 2012-2018 no se alcanzaran, es decir, no se redujo la violencia, tampoco se recuperó la paz ni disminuyeron los homicidios, secuestros y extorsiones. La falta de cumplimiento en las metas es evidente sobre todo en el tema de los homicidios dolosos (25 497 y 28809 en los dos últimos años de Gobierno de Peña Nieto) y del incremento del nivel cualitativo de violencia a partir de 2015 (Figura 2), año en el que también empezó la reducción presupuestal más pronunciada del PNPSVD, como se vio en la Figura 1. 
Figura 2. Homicidios y secuestros en México (2012-2018)

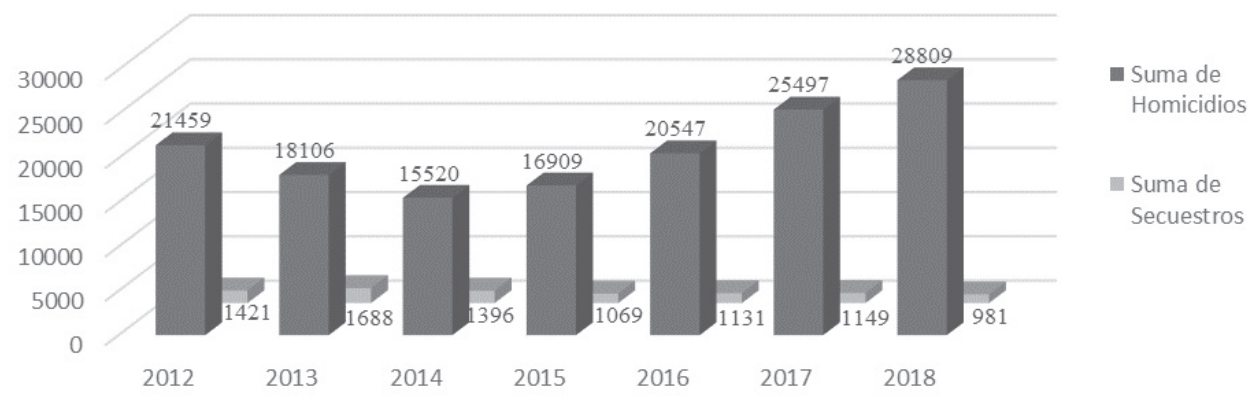

Nota: cifras por cada 100000 habitantes.

Fuente: elaboración propia con datos del Secretariado Ejecutivo del Sistema Nacional de Seguridad Pública (2019).

Además de las cifras hasta aquí descritas, debe considerarse que la percepción de inseguridad ciudadana en México aumentó considerablemente al final del sexenio. Por otro lado, las mejores cifras de dicha percepción se generaron precisamente en los años en que la política de prevención tuvo un mayor respaldo presupuestal (Figura 3).

Figura 3. Porcentaje de percepción de inseguridad en México (2012-2018)

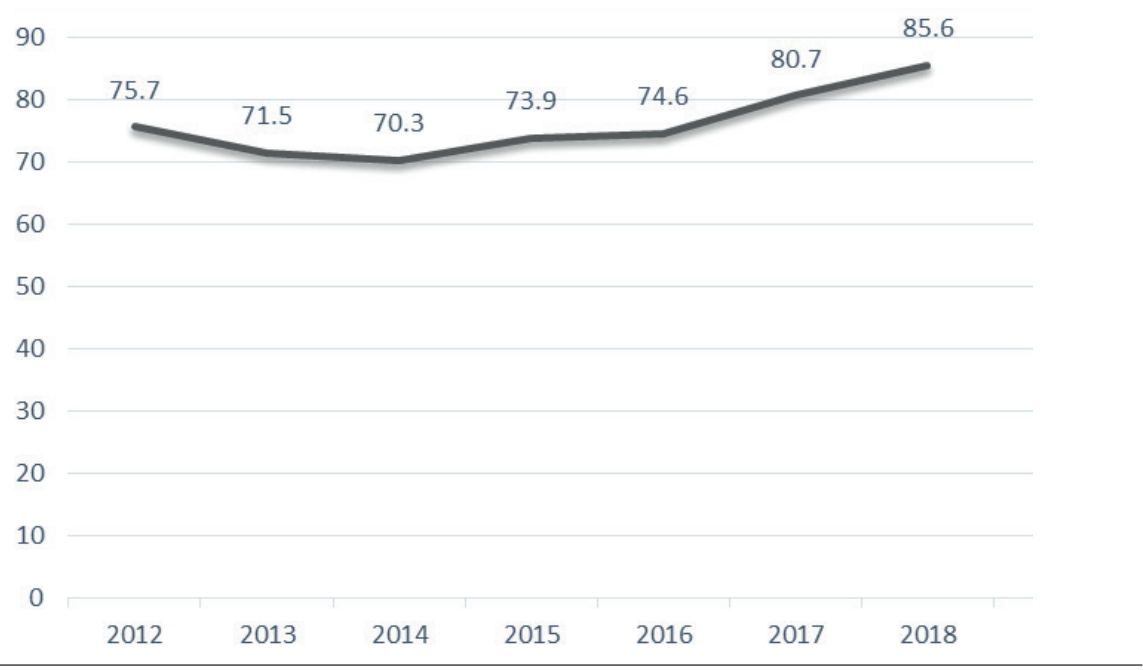

Fuente: elaboración propia con datos Instituto Nacional de Estadística y Geografía (2018a). 
Por otra parte, para 2017 el costo total de la inseguridad y el delito en hogares acumuló un monto de MXN 299.6 mil millones, es decir, $1.65 \%$ del producto interno bruto (РІB), lo cual significó un promedio de MXN 7147.00 por persona afectada (Instituto Nacional de Estadística y Geografía, 2018a, p. 27).

Por lo señalado, se puede afirmar que los objetivos prioritarios de la política de seguridad de la administración federal de Peña Nieto no se alcanzaron. Además, es importante hacer hincapié en que los resultados evidenciaron una falla en la coordinación entre agentes encargados de la implementación de la política pública. Una consecuencia de ello fue la atomización de violencia e inseguridad al final de la administración, reconocida por la propia Segob el 30 de mayo de 2018:

Esa administración federal reconoce que, contrario a la tendencia positiva registrada en sus primeros años en torno a la disminución de diversos delitos cometidos, particularmente de manera violenta en el territorio nacional, el 2017 resultó con un crecimiento inusitado en índices delictivos de alto impacto en diversas regiones del país. Lo anterior generado en un contexto de debilitamiento de algunas estructuras institucionales en distintos niveles de gobierno, sean municipales, estatales o incluso federal. (Jiménez y Rivera, 2018)

Por tal motivo, y retomando los factores que Franco Corzo (2013) plantea como determinantes de los resultados de una política pública, es pertinente señalar que no puede verse la creación e implementación de las políticas públicas como un proceso separado del contexto democrático o del tipo y calidad de la democracia del Estado donde dichas políticas buscan resolver problemas. Por ejemplo, en México, sólo el $16 \%$ de la población se siente satisfecha con la democracia, y considera que la delincuencia, la corrupción y la situación política son los principales problemas del país (Latinoba- 
rómetro, 2018, p. 23; Instituto Nacional de Estadística y Geografía, 2018b).

La democracia es, entonces, un criterio fundamental para entender por qué se diseña cierto tipo de políticas públicas y qué resultados dan estas. En este sentido, y retomando los aportes teóricos ya señalados, en los procesos democráticos que se han dado en muchos países se asume que teniendo sistemas bipartidistas o pluralistas competitivos, con sufragio universal y separación de poderes, se producirán en automático políticas públicas que sirvan a la democracia, pero estos fundamentos teóricos de las políticas públicas no se están reflejando en las democracias, incluso en las de naciones industrializadas occidentales -por ejemplo, Estados Unidos y México-, en las que se supondría que la democracia estaría más profundamente arraigada (Ingram et al., 2016, pp. 176-177).

En ese sentido, hay que agregar que el proceso de transición a la democracia que ha vivido México, aunado a la complejidad y el gran número de actores que intervienen en las políticas públicas, impacta y se refleja en el grado de control que posee el gobernante sobre el aparato estatal y la sociedad que gobierna, considerando que la ejecución de las políticas requiere de una determinada intensidad y de recursos internos y externos de coerción o de consenso, para lograr penetrar al aparato gubernamental y después llegar a la sociedad (Medellín, 2004, pp. 9-24).

Lo antes dicho es a la vez el reto y el problema que trae aparejada la descentralización vivida en países como México. Esto quiere decir, de acuerdo con Peters (2010, p. 4), que todos los Gobiernos deben delegar autoridad, dada la complejidad y el alcance de los problemas públicos que enfrentan, razón por la que gran parte de la acción del Gobierno se lleva a cabo a través de legislación delegada en la burocracia y en agencias reguladoras. 
No obstante, cuando los objetivos de las políticas públicas se desvían, también de acuerdo con Peters (2010, p. 4) y con Weimer y Vining (2005, pp. 187-188), el Estado debería tener la autoridad para retomar el control y centralizar la implementación de las políticas, como ya se había adelantado. Los autores advierten, no obstante, que en los sistemas federales el mantenimiento de la jerarquía del Estado se complejiza porque los Gobiernos subnacionales, que disfrutan de una discreción considerable sobre la política y la administración, apelan a la autonomía.

Por esto es que, desde el nuevo institucionalismo, se menciona que los individuos importan lo mismo que las reglas, o incluso más, pues garantizan su cumplimiento, lo que es la medida de éxito de toda política pública (Merino, 2013, p. 66). Cuando se hace alusión a la importancia de los individuos sobre las instituciones, se está en realidad trayendo a colación que los intereses particulares generalmente están por encima de los colectivos, como ya se esbozó. En este punto, se puede apreciar la dicotomía existente en el actuar de los agentes encargados de la implementación de políticas como el PNPSVD, sobre todo porque no todas las políticas (lo mismo en el caso de las reformas estructurales) parecen tener el mismo grado de urgencia o los mismos costos en su instrumentación.

Ese peso de ciertas figuras particulares en el éxito de las políticas públicas lo reafirmó el propio Ejecutivo federal bajo análisis ante la Conferencia Nacional de Gobernadores (Conago) en el último año de su Gobierno (2018):

[...] Hace cinco años[,] durante mi primera participación en este foro[,] asumí el compromiso de trabajar junto con cada uno de los miembros de esta conferencia para promover una profunda transformación nacional[;] propuse en aquel momento impulsar un federalismo articulado que generara sinergias entre los tres órdenes de gobierno. El trabajo conjunto ha dado resultados, la agenda de las reformas estructurales que 
llevamos a cabo no habría sido posible sin el respaldo de las principales fuerzas políticas representadas en el Congreso o sin el apoyo de los Gobiernos y de las legislaturas estatales. (Sánchez, 2018)

Es necesario señalar que la cooperación y la coordinación entre órdenes de gobierno funcionaron para la aprobación de las políticas (corto plazo), pero con las políticas que requerían mayor impulso, y por ende mayores costos por la urgencia de resultados, como las del PNPSVD, los agentes implementadores parecieron dejar de lado la retórica del federalismo articulado transmitida por el presidente y hacer que el Gobierno federal asumiera los costos políticos.

La postura de los agentes, en este caso los gobernadores, también recuerda que con la descentralización política los Gobiernos subnacionales han obtenido capacidad para ejercer el control político y económico de manera discrecional en sus territorios. Asimismo, el concepto de la descentralización también se presenta cuando se observa cómo se ejecutaron las aportaciones y participaciones federales a los estados en el periodo peñanietista, lo que refleja que el Gobierno central induciría la cooperación mediante recompensas que aseguren la participación de las administraciones subnacionales en la instrumentación de las políticas que impulsa (Figura 4). 
Figura 4. Participaciones y aportaciones federales a los estados (2012-2017) (cifras en miles de millones de pesos)

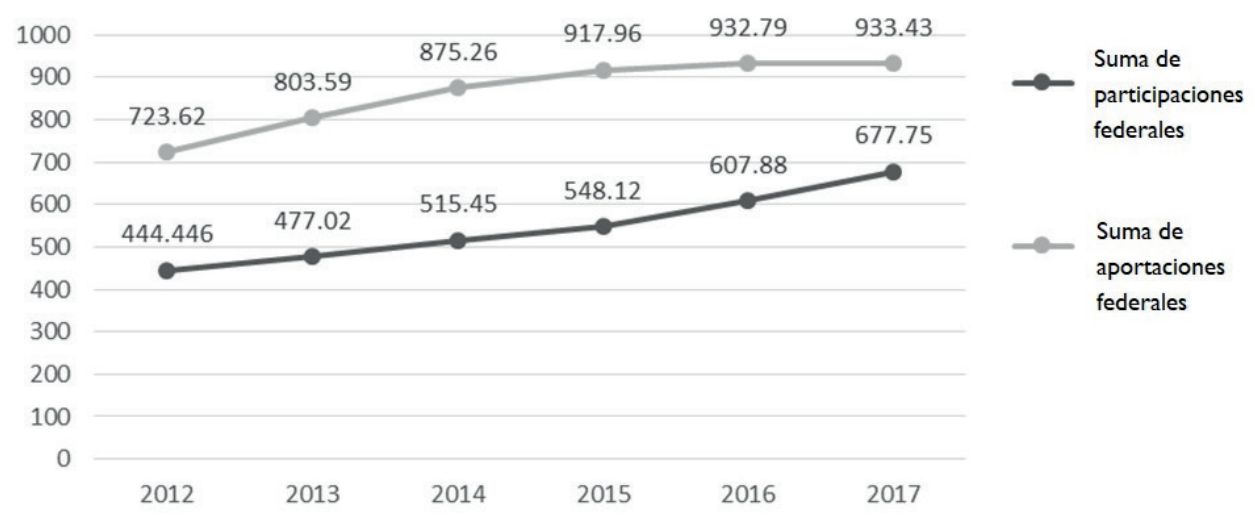

Fuente: elaboración propia con datos de Instituto Nacional de Estadística y Geografía (2019).

Ahora bien, la acumulación del poder político y económico en los Gobiernos subnacionales durante el sexenio de Peña Nieto no significó una mejora en las condiciones de seguridad en el país, y sí se dio al parejo de un aumento en el poder bélico de las organizaciones criminales contra el Estado mexicano. Esto, por un lado, mostró las fallas de gobierno y, por otro lado, la falta de institucionalización del orden y la pérdida territorial en varias entidades del país.

Por ejemplo, en 2014 en el estado de Michoacán, ante el grado de urgencia y de ingobernabilidad ocasionado por el enfrentamiento entre el grupo criminal Los Caballeros Templarios y los grupos de autodefensas, el Gobierno federal nombró - desapareciendo la figura del gobernador-a un comisionado para la Seguridad y el Desarrollo Integral, Alfredo Castillo Cervantes (Diario Oficial de la Federación, 2014c).

Dos ejemplos más: el 26 de septiembre de 2014, cuarenta y tres estudiantes de la escuela normal de Ayotzinapa, en Guerrero, fueron atacados por policías municipales de Iguala y Cocula, entregados al grupo criminal Guerreros Unidos y luego desaparecidos; el 1 de mayo de 2015, el grupo 
criminal Cártel Jalisco Nueva Generación (CJNG), durante una persecución en Jalisco, derribó un helicóptero de la Secretaría de la Defensa Nacional (Sedena), en medio de una oleada de ataques que incluyó narcobloqueos, enfrentamientos e incendios de gasolineras y bancos en todo el estado.

El panorama anterior refleja los tres grandes problemas teóricos -los problemas inherentes al suministro burocrático y a la implementación y la descentralización- a los que se enfrentó la política de prevención social de la violencia y delincuencia peñanietista, pero el problema de la descentralización y las complejidades que trajo aparejadas no sólo se vincularon con la violencia y la inseguridad, sino también con la evidencia de intereses particulares que parecieron motivar a los gobernadores. Así, durante el periodo 2012 2018, fueron procesados al menos once exgobernadores ${ }^{6}$ por los delitos de corrupción, lavado de dinero, fraude, asociación delictiva, delincuencia organizada, defraudación fiscal, delitos contra la salud y peculado agravado, entre otros (García, 2018).

Dicho esto, las fallas de gobierno son entonces el verdadero punto de partida para el reto de hacer frente a la inseguridad en el país, lo que se suma a la insatisfacción general con la implementación de las políticas públicas en la materia.

Con el arribo del Gobierno federal sucesor de Peña Nieto, encabezado por el presidente Andrés Manuel López Obrador (2018-2024), la respuesta al problema de la inseguridad ha sido centralizar en un solo cuerpo de élite, la Guardia Nacional, el combate a la inseguridad, la violencia y las organizaciones criminales. De esta manera, la Guardia Nacional es el último recurso en materia de seguridad

6. César Duarte (Chihuahua), Jesús Reyna (Michoacán), Guillermo Padrés (Sonora), Andrés Granier Melo (Tabasco), Javier Duarte de Ochoa (Veracruz), Eugenio Hernández Flores (Tamaulipas), Tomás Yarrington (Tamaulipas), Roberto Borge Angulo (Quintana Roo), Luis Armando Reynoso Femat (Aguascalientes), Flavino Ríos (Veracruz) y Rodrigo Medina (Nuevo León). 
pública del Estado para centralizar y recuperar el control territorial en zonas capturadas por el crimen organizado.

La creación de la Guardia Nacional refleja al menos dos problemas institucionales. El primero es que no se ha fortalecido la coordinación entre órdenes de gobierno ni se ha planteado el desarrollo y consolidación de las policías estatales y municipales, y en cambio sí se ha ejecutado la desaparición de instituciones como la Policía Federal. El segundo problema es que la Guardia Nacional muestra los retrocesos y claroscuros de la democracia mexicana en su búsqueda por brindar un adecuado Estado de derecho, lo cual ha ocasionado la legitimación social de medidas extraordinarias como la militarización de la seguridad pública.

De esta manera, puede decirse que la estrategia del Gobierno de López Obrador es, ante un contexto atomizado, la de centralizar el mando de la seguridad en una sola corporación. No obstante, los retos a corto y largo plazo para dicha nueva estrategia podrían ser más costosos, ello debido a que no queda claro el grado de responsabilidad de los agentes ejecutores, como los gobernadores, para actuar en contra de la violencia e inseguridad en sus estados.

Incluso, desde la postura de Weimer y Vining (2005, p. 187), "la descentralización tiende a obstaculizar la aplicación de las políticas [...], si bien la descentralización es deseable, no es una esencial característica estructural del Gobierno, debido a que a veces limita la eficacia de las políticas públicas". Esto no significa que deba apostarse por la centralización del Estado, pero es fundamental comprender que un Estado debe tener la fortaleza institucional para involucrar a los agentes que intervienen en la implementación de políticas públicas.

Lo dicho hasta aquí ayuda a entender que el análisis de una política pública no puede reducirse únicamente a aspectos metodológicos y técnicos. Es fundamental considerar el 
nuevo panorama sociopolítico en México, sobre todo cuando este tipo de medidas se dan en contextos democráticos, es decir, que en gran medida tienen apoyo y legitimación de la sociedad. De ahí que se abran nuevas pautas de estudio que vinculen las consecuencias de la transición a la democracia en el diseño y ejecución de políticas públicas como el PNPSVD.

\section{Conclusiones}

En el presente artículo, se analizó la estrategia principal de la política de seguridad del Gobierno encabezado por el presidente Enrique Peña Nieto (2012-2018), la cual se basó en la prevención del delito. El estudio partió de interrogarse por qué si la política planteada por la administración federal peñanietista fue distinta a la de su antecesora, la violencia y la inseguridad se atomizaron en el país y se alcanzaron registros históricos en el nivel cualitativo de dicha violencia hacia el final del Gobierno. Se ha expuesto que la política de seguridad, que tuvo como referente al PNPSVD, falló por los problemas inherentes al suministro burocrático, lo que conllevó problemas de implementación que a su vez se complejizaron con el contexto de descentralización política que vive el país desde su democratización reciente.

Los referentes teóricos del nuevo institucionalismo y de las fallas de gobierno fueron utilizados para respaldar los hallazgos y el análisis realizado, con miras a la comprobación de la hipótesis de trabajo. Se expuso que una política pública es la respuesta del Estado a una demanda de la sociedad o su anticipación a un problema, pero que para tener resultados positivos de la misma se requiere que los individuos que controlan las instituciones converjan con los intereses y objetivos de la política en cuestión.

También se precisó cómo, no obstante, el comportamiento de los individuos termina siendo más determinante que la propia creación de las instituciones (considerando 
como tales a las políticas públicas). Así, el PNPSVD, si bien inició impulsado por un presupuesto para cumplir con los objetivos, fue acusando una disminución en el mismo que evidenció que los intereses del Gobierno federal de Peña Nieto estaban en la agenda económica y no en reducir las causas de la violencia e inseguridad en el país. Aunado a esto, los cambios del titular de la Subsecretaría de Prevención y Participación Ciudadana respondieron más a cuestiones políticas que a la búsqueda de fortalecimiento de los objetivos de la política de prevención que se estaba implementando.

La consecuencia principal de estas fallas en la política fue el incremento de la violencia e inseguridad, así como una renuencia o desinterés por parte de los Gobiernos estatales para asumir la parte de responsabilidad que les correspondía para hacer frente al fenómeno delictivo. Este punto ha evidenciado un problema que ha venido aparejado con la llegada de la democracia, es decir, que los Gobiernos tienen que delegar funciones a los demás actores para cumplir con los objetivos de las políticas, lo cual, si se tiene un Estado frágil institucionalmente y con severas pérdidas del control territorial -como sucede en Guerrero, Michoacán, Guanajuato, Veracruz y Tamaulipas-, hace complejo instrumentar una política pública integral en países como México.

Por último, el análisis realizado demostró que el uso de estrategias retóricas por los instrumentadores de las políticas públicas generalmente tiene beneficios a corto plazo, aunque las consecuencias a largo plazo terminan siendo asumidas por el resto de la sociedad.

Acemoglu, D., y Robinson, J.A. (20/3). Por qué fracasan los

Bibliografía países. Los origenes del poder, la prosperidad y la pobreza. México: Crítica.

Agudo Sanchíz,A. (20 I 5). La vida social de los documentos de las políticas públicas. Nueva antropología, xxVIII(83), I23-146. 
Agudo Sanchíz, A. (2017). Reorganización del Gobierno mediante la sociedad civil. El papel de los actores privados en las políticas de prevención de la violencia. Madrid: Ediciones Complutense.

Buchanan, J. M., y Tullock, G. (1993). El cálculo del consenso. Fundamentos lógicos de la democracia constitucional. Obras maestras del pensamiento contemporáneo. España: Planeta D'Agostini.

Cano, l.,y Rojido, E. (2016). Mapeo de programas de prevención de homicidios en América Latina y el Caribe. Río de Janeiro: Laboratorio de Análisis de la Violencia y Foro Brasileño de Seguridad Pública.

Cámara de Diputados (20I5). LXII legislatura. México. Recuperado de sitl.diputados.gob.mx/LXII_leg/info_diputados. php

Cámara de Senadores (20I5). Senadores integrantes de la LXII y LXIII legislaturas. Recuperado de http://www.senado.gob. $m x /$ ?ver=int\& $m n=4 \& s m=5$

Colomer, J. M. (199I). Lecturas de teoría política positiva. Madrid: Instituto de Estudios Fiscales, Ministerio de Economía y Hacienda.

Diario Oficial de la Federación (2013). Plan Nacional de Desarrollo 2013-2018. Recuperado de http://www. dof.gob.mx/nota_detalle.php?codigo $=5299465 \&$ fecha $=20 / 05 / 2013$

Diario Oficial de la Federación (20I4a). Programa Nacional para la Prevención Social de la Violencia y Delincuencia 2014-2018. Recuperado de http://www.dof.gob.mx/ nota_detalle.php?codigo $=5343087 \&$ fecha $=30 / 04 / 2014$ Diario Oficial de la Federación (20।4b). Acuerdo por el que se establecen las tarifas autorizadas para el ejercicio fiscal 2014, para el pago de servicios de protección, custodia, vigilancia y seguridad de bienes, instalaciones. Recuperado de https://www.dof.gob.mx/nota_detalle. php? codigo $=5346583 \&$ fecha $=29 / 05 / 2014$ 
Diario Oficial de la Federación (20I4c). Decreto por el que se Bibliografía crea la Comisión para la Seguridad y el Desarrollo Integral en el estado de Michoacán. Recuperado de http:// www.dof.gob.mx/nota_detalle.php?codigo $=5329743 \&$ fecha $=|5 / 0| / 20 \mid 4$

Franco Corzo, J. (2013). Diseño de políticas públicas. México: IEXE Editorial.

García, D. (2018). Borge, uno más a la lista de exgobernadores procesados. El Universal. Recuperado de http://www.eluniversal.com.mx/nacion/seguridad/borge-uno-mas-la-lista-de-ex-gobernadores-procesados

Garson, G. D. (2007). De la ciencia de políticas al análisis de políticas: veinticinco años de progreso. En L. F. Aguilar Villanueva (Ed.), El estudio de las políticas públicas (pp. 149-180). México: Miguel Ángel Porrúa.

Hernández, G. (20I5). Análisis de la política de seguridad en México: 2006-2012. Política y cultura, (44), I59-I87. Recuperado de http://www.scielo.org.mx/pdf/polcul/ n44/n44a8.pdf

Hernández, J.J. (20I2). Lucha a narco que convenga, no que complazca a la DEA: EPN. El Universal. Recuperado de https://archivo.eluniversal.com.mx/notas/85773 I.html

Ingram, H., De León, P., y Schneider, A. (2016). Public Policy Theory and Democracy:The Elephant in the Corner. En B. G. Peters, y P.Zittoun (Eds.), Contemporary Approaches to Public Policy.Theories, Controversies and Perspectives (pp. 175-200). Londres: Palgrave Macmillan.

Instituto Mexicano de Competitividad (2015). Preocupa a sociedad civil el nombramiento de Escobar como subsecretario de Prevención y Participación Ciudadana. Recuperado de https://imco.org.mx/politica_buen_gobierno/ preocupa-a-sociedad-civil-el-nombramiento-de-escobar-como-subsecretario-de-prevencion-y-participacion-ciudadana/ 
Bibliografía Instituto Nacional de Estadística y Geografía (20I5). Información nacional, por entidad federativa y municipios. Recuperado de https://www.inegi.org.mx/app/areasgeograficas/ Instituto Nacional de Estadística y Geografía (20l8a). Encuesta Nacional deVictimización y Percepción sobre Seguridad Pública 2012-20 I7. Recuperado de https://www. inegi.org.mx/programas/envipe/20 I /inegi.org.mx/contenidos/programas/envipe/2017/doc/envipe2017_presentacion_nacional.pdf

Instituto Nacional de Estadística y Geografía (20l8b). Encuesta Nacional de Seguridad Pública Urbana 2018. Recuperado de https:/www.inegi.org.mx/contenidos/ saladeprensa/boletines/20I8/ensu/ensu20 I8_I0.pdf

Instituto Nacional de Estadística y Geografía (2019). Estadística de finanzas públicas estatales y municipales. Recuperado de http://www.inegi.org.mx/sistemas/mexicocifras/ default.aspx? $\mathrm{e}=23$

Instituto para la Economía y la Paz (20I5). Índice de paz 2015. Un análisis de la dinámica de los niveles de paz en México. Ciudad de México: Organización de las Naciones Unidas. Jiménez, H.,y Rivera,A. (2018).Segob reconoce incremento de delitos de alto impacto.EI Universal. Recuperado de http:// www.eluniversal.com.mx/nacion/seguridad/segob-reconoce-incremento-de-delitos-de-alto-impacto\# Lasswell, H. D. (2007). La orientación hacia las políticas. En L. F. Aguilar Villanueva (Ed.), El estudio de las políticas públicas (pp. 120-150). México: Miguel Ángel Porrúa. Latinobarómetro (2018). Latinobarómetro informe 2018. Santiago de Chile: Corporación Latinobarómetro. Leal, E. (I8 de junio de 20I5). Entrevista personal en su calidad de coordinador de asesores de la Subsecretaría de Prevención y Participación Ciudadana [G. Hernández Hernández, entrevistador]. Ciudad de México, México. Leyva, A. (2017). Qué importa más en la aprobación presidencial de Peña Nieto. Animal político. Recuperado 
de https://www.animalpolitico.com/blogueros-lecciones-de-las-elecciones/201 7/ I I/I7/importa-mas-la-aprobacion-presidencial-pena-nieto/

Lindblom, C. (2007). La ciencia de salir del paso. En L. F. Aguilar Villanueva (Ed.), La hechura de las políticas (pp. 203-245). México: Miguel Ángel Porrúa.

López Sandoval, I. (2007). La rentabilidad política electoral del gasto social en el sistema político mexicano durante el periodo 1994-2006 [tesis de doctorado inédita]. Facultad Latinoamericana de Ciencias Sociales: México.

López Sandoval, I. (2010). Racionalidad individual y construcción de reglas en el diseño de las constituciones políticas de 1824, 1857 y 1917. En A. Gimate-Welsh, y P. Castro (Coords.), Sistema político mexicano ayer y hoy, continuidades y rupturas (pp. 300-320). México: Porrúa.

Medellín, P. (2004). La política de las políticas públicas:propuestas teórica y metodológica para el estudio de las políticas públicas en países de frágil institucionalidad. Serie Políticas sociales. Santiago de Chile: CEPAL. Recuperado de https:// repositorio.cepal.org/bitstream/handle/ I I 362/6082/ I/ S047566_es.pdf

Méndez, E. (2016). Alberto Begné, nuevo subsecretario de Prevención y Participación Ciudadana. Excélsior. Recuperado de https://www.excelsior.com.mx/nacio$\mathrm{nal} / 2016 / 02 / 25 / 1077405$

Merino, M. (2013). Políticas públicas. Ensayo sobre la intervención del Estado en la solución de problemas públicos. México: CIDE.

North, D. (1993). Instituciones, cambio institucional y desempeño económico. México: Fondo de Cultura Económica.

Parsons, W. (2007). Políticas públicas. Una introducción a la teoría y la práctica del análisis de políticas públicas. México: Flacso-México y Miño y Dávila Editores.

Pasquino, G. (20I I). Nuevo curso de ciencia política. México: Fondo de Cultura Económica. 
Bibliografía $\quad$ Pérez Sánchez, M. (2005). El estudio de las políticas públicas. En M. Pérez Sánchez (Ed.), Análisis de políticas públicas (pp. 3 I-50). España: Editorial Universidad de Granada.

Peters, G. (20I0). Governing in the shadows. Alemania: Research Center.

Presidencia de la República (2013). Discurso por el 85 Aniversario y Día del Policía Federal. I 3 de julio de 2013. Recuperado de https://www.gob.mx/presidencia/ prensa/diversas-intervenciones-durante-el-85-aniversario-y-dia-del-policia-federal

Presidencia de la República (2015a). Política pública de seguridad y procuración de justicia. Recuperado de http://www. presidencia.gob.mx/politicadeseguridad/

Presidencia de la República (20 I 5b). Discurso por la publicación de la Ley General de Víctimas. Recuperado de https://www. gob.mx/presidencia/prensa/diversas-intervenciones-durante-la-publicacion-de-la-ley-general-de-victimas

Real Dato, J. (2005). Actores, ideas e instituciones en las políticas públicas. En M. Pérez Sánchez (Ed.), Análisis de políticas públicas (pp. 77-I07). España: Editorial Universidad de Granada.

Romero, J.J. (1999). Los nuevos institucionalismos: sus diferencias, sus cercanías. En W.W. Powell, y P. J. Dimagio (Comps.), El nuevo institucionalismo en el análisis organizacional (pp. 7-75). México: Fondo de Cultura Económica. Ruiz, F. (20I5). Ejército y Marina llegan a Chalco y Tláhuac. El Universal. Recuperado de https://www.eluniversal. com.mx/articulo/metropoli/20I5/05/28/ejercito-y-marina-Ilegan-chalco-y-tlahuac\#imagen- I

Sánchez, E. (20I8). Peña convoca a gobernadores a redoblar esfuerzos en materia de seguridad. Excélsior. Recuperado de http://www.excelsior.com.mx/nacional/pena-convoca-a-gobernadores-a-redoblar-esfuerzos-en-materia-de-seguridad/I240546\#

\section{8}


Secretaría de Gobernación (2013). Bases del Programa NacioBibliografía nal para la Prevención Social de laViolencia y Delincuencia e Instalación de la Comisión Intersecretarial. México: Secretaría de Gobernación.

Secretariado Ejecutivo del Sistema Nacional de Seguridad Pública (2019). Incidencia delictiva del fuero común 20I220 /7. Recuperado de http://secretariadoejecutivo.gob. $\mathrm{mx} /$ incidencia-delictiva/incidencia-delictiva-fuero-comun.php

Transparencia Presupuestaria (2018). Programa Nacional de Prevención del Delito. Recuperado de http://nptp.hacienda. gob.mx/programas/jsp/programas/fichaPrograma.jsp?ciclo $=2016 \&$ id $=04 U 006$

Weimer, D. L., y Vining, A. R. (2005). Policy Analysis Concepts and Practice. Englewood Cliffs: Prentice Hall. 